\title{
La prescripción de la ganancia en la adaptación audioprotésica
}

\author{
Franz Zenker
}

Para citar este artículo:

Zenker F. (2002). La prescripción de la ganancia en la adaptación audioprotésica. Auditio, 1(3), 45-52. https://doi.org/10.51445/sja.auditio.vol1.2002.0018

Enlace al artículo:

https://doi.org/10.51445/sja.auditio.vol1.2002.0018

Historial:

Publicado (online): 01-10-2002 


\author{
Franz Zenker \\ Clínica Barajas. \\ Santa Cruz de Tenerife. España.
}

\begin{abstract}
Resumen
Los audífonos basados en la tecnología digital han fomentado la aparición de nuevas fórmulas de prescripción de la ganancia. En la actualidad existen dos grandes grupos de fórmulas: las genéricas, aplicables a cualquier tipo de audífono, como el DSL o el NAL-R y las fórmulas específicas desarrolladas por los fabricantes para modelo y marcas concretas de audífonos, como el ASAll de Oticon o el DIGITAL de Phonak. Ambos grupos de fórmulas poseen ventajas y limitaciones. Las fórmulas específicas son rápidas y no requieren grandes conocimientos. La fórmulas genéricas, por otro lado, permiten considerar de una forma amplia los aspectos diferenciadores de cada individuo en la prescripción final de la ganancia. En este artículo revisamos los principales métodos de prescripción de la ganancia genéricos al uso aparecidos en los últimos años.
\end{abstract}

Palabras Claves: audioprótesis, adaptación, prescripción de la ganancia, NAL, DSL, IHAFF, POGO, FIG6

\section{Introducción}

La rehabilitación de la discapacidad auditiva mediante amplificación conlleva una serie de pasos que implica la toma de decisiones secuenciales que determinarán el rendimiento final. En todo este proceso, la prescripción de la ganancia en función de la evaluación audiológica resulta crítica. Con la aparición de las prótesis auditivas digitales y programables el proceso de prescripción se integra de forma automática en el software de adaptación perdiendo protagonismo las decisiones del audioprotesista. Mediante la automatización del juicio del experto la prescripción de la ganancia se limita a la introducción de los valores de la audiometría en el software de adaptación. El error que cabe esperar en este tipo de adaptaciones es proporcional a la diferencia de las características individuales del paciente respecto a la media de referencia establecida por el fabricante. Los métodos de prescripción de la ganancia genéricos tienen en cuenta en mayor medida los aspectos diferenciadores que pueden maximizar el beneficio de la adaptación en poco tiempo.

Durante los últimos veinte años se ha discutido la eficacia de los métodos de prescripción de la ganancia dado que su utilidad no quedaba clara ante los pobres resultados de las adaptaciones audioprotésicas en general (1). En los años cuarenta la amplificación se establecía de una forma puramente intuitiva. Los aparatos disponibles no permitían grandes ajustes limitándose en la mayoría de los casos a amplificar el sonido sin tener en cuenta las características audiológicas de los pacientes. Carhart (1) en 1946 fue el primero en tratar de racionalizar el proceso de adaptación estableciendo relaciones entre distintos tipos de audífonos y la aceptación de los mismos por parte de los usuarios. Watson y Knudsen (2) y posteriormente Lybarger (3) continuarion con trabajos clásicos en los que se establecia la ganancia del audífono en función de los datos audiométricos. Sin embargo durante los años cuarenta se abandona esta perspectiva a partir de los resultados obtenidos con el Harvard Report (4). A partir de este informe se adopta una metodología basada en la selección del audífono en función de las comparación establecidas por los pacientes entre aparatos (5). No es hasta las dos últimas décadas en las que se comienza a retomar el interés por las fórmulas de prescripción de la ganancia (6). Estos métodos prescriptivos implican el uso de un procedimiento de cálculo y fórmulación matemática con objeto de predecir las características de amplificación que resultan mas adecuadas para un perfil audiológico determinado.

Cornelisse et al. definió la prescripción de la ganancia como "Una función que prescribe la ganancia del audífonos de forma específica en frecuencia en base a los valores audiométricos del paciente" (7). Hawkins (8) propuso una clasificación en dos categorías para las fórmulas de prescripción en función de la información utilizada en él calculo. En un primer 
grupo clasificó aquellas fórmulas que utilizan únicamente la información del umbral auditivo del paciente. En segundo lugar las fórmulas que incorporan información supraliminar. Sin embargo, en opinión de Bryne (9) está clasificación no resulta crítica ya que los requisitos de ganancia y respuesta frecuencial pueden ser predichos tanto a partir de medidas liminares como supraliminares. La bondad de la prescripción estará más en la capacidad de las fórmulas que en los datos audiológicos ya que estas son en último término los que reflejan con mayor o menor exactitud las relaciones específicas entre las variables obtenidas a partir de la evaluación y los parámetros finales de amplificación.

En general, todos los acercamientos basados en la prescripción de la ganancia mediante algún tipo de cálculo o formulación tienen los siguientes objetivos comunes (10):

1. Prescribir la ganancia apropiada para alcanzar el umbral funcional cercano a la audición normal.

2. Prescribir un volumen confortable para el promedio del espectro del habla.

3. Prescribir la máxima ganancia dentro del rango dinámico.

4. Prescribir parámetros que restauren la sensación de sonoridad.

5. Prescribir el habla amplificada al nivel de máximo confort dentro de las frecuencias del habla.

6. Prescribir la ganancia teniendo en cuenta el umbral de inconfort.

A su vez, los métodos prescriptivos pueden ser clasificados en función del tipo de aparato para el cual han sido diseñado. En el momento actual podemos distinguir entre los audífonos lineales y no-lineales. Con la aparición de los audífonos capaces de comprimir la señal en bandas de forma independiente surgen los métodos de prescripción capaces de calcular la ganancia de salida de forma específica en frecuencia. En la Tabla I se observa la clasificación de los métodos al uso en función de la tecnología del audífono y los parámetros de entrada (11).

Los audífonos no-lineales requieren de la prescripción de parámetros como el ratio y umbral de compresión. Estos parámetros no pueden determinarse únicamente sobre la base de un solo valor de referencia como ocurre con los amplificadores lineales. La adaptación de audífonos no-lineales conlleva él calculo de diferentes valores de ganancia en función de diferentes valores de entrada.

\section{Métodos de prescripción de la ganancia para audífonos lineales}

a) National Acoustic Laboratory (NAL-R). El NAL fue descrito en su primera fórmulación por Byrne y Tonnisson (24) y posteriormente fue revisado por Byrne y Dillon (13). Esta revisión fue llevada acabo en un intento por prescribir una respuesta en frecuencia optima que facilitase al máximo la inteligibilidad del habla después de que el control del volumen del audífono hubiese sido ajustado al nivel preferido por el usuario (13). La optimización del espectro del habla implica un intento para maximizar la inteligibilidad sobre una amplio rango de frecuencia. La idea a partir de la cual parte el NAL-R es alcanzar este objetivo en la medida en la que todas las bandas de frecuencia del habla contribuyan de igual forma en la sonoridad de la señal.

En general siempre ha existido una tendencia a prescribir poca ganancia en bajas frecuencias en relación con la ganancia prescrita en frecuencias medias y altas. Así mismo, las propuestas de ganancia para aplicar en función de la pendiente del audiograma varían en función del método propuesto (13).

Tabla I: Principales métodos de prescripción de la ganancia.

\begin{tabular}{|c|c|c|c|}
\hline Regla & Autor & Parámetros de entrada & Audífono \\
\hline 1/2 Ganancia & Berger et al. (6) & HTL & Lineal \\
\hline POGO & McCandless y Lyregaard (12) & HTL, UCL & Lineal \\
\hline NAL-R & Byrne y Dillon (13) & HTL & Lineal \\
\hline Fig. 6 & Killion y Fikret-Pasa (14) & HTL & No-lineal \\
\hline DSL[i/o] & Seewald (15) & HTL,UCL & No-lineal \\
\hline NAL-NL1 & Dillon (16) & HTL,UCL & No-lineal \\
\hline IHAFF & Allen et al. (17) & Función de sonoridad & No-lineal \\
\hline Scal Adapt & Kiessling et al. (18) & Sonoridad amplificada por bandas del AGC & No-lineal \\
\hline A-life 9000 & Haubold (19) & 6 dimensiones de calidad del sonido & No-lineal \\
\hline Cambridge & Moore et al. (20) & $\begin{array}{l}\text { HTL + sonoridad del habla amplificada + ajuste } \\
\text { de sonoridad }\end{array}$ & No-lineal \\
\hline CascAdapt & Pastoors et al. (21) & $\begin{array}{l}\text { Sonoridad amplificada por bandas del AGC + } \\
\text { ajuste sonoridad }\end{array}$ & No-lineal \\
\hline Logic & ReSound & HTL,UCL & No-lineal \\
\hline Digital/Ski-Slope & Phonak & HTL,UCL & No-lineal \\
\hline ASA2/Ski & Oticon - Schum (22) & HTL,UCL & No-lineal \\
\hline Loudnes mapping & Widex - Ludvigsen (23) & HTL,UCL & No-lineal \\
\hline
\end{tabular}


Para corregir estas dificultades y añadir una mayor utilidad al procedimiento NAL, Byrne y Dillion (13) propusieron el NAL-R. Este procedimiento optimiza la energía disponible para determinadas perdidas auditivas en todas las frecuencias del habla, particularmente en las bandas de bajas frecuencias entre los $500 \mathrm{~Hz}$ y $1000 \mathrm{~Hz}$. Por otro lado, el NAL-R sustituye la regla de la mitad de la ganancia, sobre el que había sido desarrollado, por la de un tercio de la ganancia e introduce el promedio de las frecuencias $500 \mathrm{~Hz}, 1 \mathrm{kHz}$ y $2 \mathrm{kHz}$ lo cual incrementa a un ratio de un $46 \%$ la ganancia en función de la media de estos valores.

Tabla II: Fórmula del NAL-R para el cálculo del REIG propuesta por Byrne y Dilion (13)

\begin{tabular}{ccccc}
\hline \hline 250 & $\mathrm{X}+0,31$ & $\mathrm{HL} @ 250$ & -17 & $=$ REIG \\
500 & $\mathrm{X}+0,31$ & $\mathrm{HL} @ 500$ & -8 & $=$ REIG \\
1000 & $\mathrm{X}+0,31$ & $\mathrm{HL} @ 1000$ & -3 & $=$ REIG \\
1500 & $\mathrm{X}+0,31$ & $\mathrm{HL} @ 1500$ & +1 & $=$ REIG \\
2000 & $\mathrm{X}+0,31$ & $\mathrm{HL} @ 2000$ & +1 & $=$ REIG \\
3000 & $\mathrm{X}+0,31$ & $\mathrm{HL} @ 3000$ & -1 & $=$ REIG \\
4000 & $\mathrm{X}+0,31$ & $\mathrm{HL} @ 4000$ & -2 & $=$ REIG \\
6000 & $\mathrm{X}+0,31$ & $\mathrm{HL} @ 6000$ & -2 & $=$ REIG \\
\hline $\mathrm{X}=0.5(\mathrm{HL} @ 500+\mathrm{HL} @ 1000+\mathrm{HL} @ 2000)$ \\
\hline
\end{tabular}

En la Tabla II podemos observar la fórmula del NAL-R para el cálculo de la ganancia de inserción en oído real (REIG). El valor de X en esta fórmula es el producto de multiplicar la constante 0,005 por la suma de los umbrales tonales a $500 \mathrm{~Hz}, 1000 \mathrm{~Hz}$ y $2000 \mathrm{~Hz}, \mathrm{Y}$ es una constante con valor 0,31 y Z es un factor de corrección que varía para cada frecuencia según se trate de un audífono retroauricular, intrauricular o intracanl, así como según se realice las medidas en acoplador de 2cc o en simulador de oído. La fórmula del NAL incluye $10 \mathrm{~dB}$ de ganancia de reserva (25).

Tabla III: Modificaciones sobre el NAL-R para pérdidas severas y profundas $(26,27)$.

Modificación 1

Sí X para $500 \mathrm{~Hz}, 1 \mathrm{kHz}$ y $2 \mathrm{kHz}$ excede 180 entonces: sumar $0.116(X-180)$ a $X$ en la que $X=$ Suma total del HL@500, 1000 y 2000

Modificación 2

Ajustes del NAL-R para pérdidas que excedan lo 90 d a $2 \mathrm{kHz}$

\begin{tabular}{cccccccccc}
\hline \multicolumn{1}{l}{ Pérdida } & $\mathbf{c} 2 \mathbf{k H z}$ & \multicolumn{10}{c}{ Frecuencia en $\mathbf{~ H z}$} \\
\hline $\mathrm{dB}$ HL & .25 & .5 & .75 & 1 & 1.5 & 2 & 3 & 4 & 6 \\
95 & 4 & 3 & 1 & 0 & -1 & -2 & -2 & -2 & -2 \\
100 & 6 & 4 & 2 & 0 & -2 & -3 & -3 & -3 & -3 \\
105 & 8 & 5 & 2 & 0 & -3 & -5 & -5 & -5 & -5 \\
110 & 11 & 7 & 3 & 0 & -3 & -6 & -6 & -6 & -6 \\
115 & 13 & 8 & 4 & 0 & -4 & -8 & -8 & -8 & -8 \\
120 & 15 & 9 & 4 & 0 & -5 & -9 & -9 & -9 & -9 \\
\hline \hline
\end{tabular}

En el NAL-R se llevaron acabo dos modificaciones adicionales que podemos observar en la Tabla III en orden a adaptar este procedimiento a pacientes con pérdidas auditivas severas $(26,27)$. La primera modificación es un incremento en el factor $\mathrm{X}$ en caso de que la media en las tres frecuencias $(500 \mathrm{~Hz}, 1000$ $\mathrm{Hz}$ y $2000 \mathrm{~Hz}$ ) exceda los $60 \mathrm{~dB}(8)$. La segunda modificación aumenta la ganancia en las bajas frecuencias y reduce las altas en caso de que el umbral a $2000 \mathrm{~Hz}$ exceda los $90 \mathrm{~dB}$. Estos ajustes modifican la respuesta del audífono en las regiones de altas y bajas frecuencias en orden a facilitar la adaptación del audífono a pacientes con perdidas auditivas severa. Este tipo de pacientes necesita mas energía en las bajas frecuencias y menos en las altas en orden a reducir los problemas derivados de la retroalimentación del audífono.

\section{b) Presciption Of Gain And Output (POGO)}

El método de prescripción de la ganancia POGO se basa en las preferencias manifestadas por los usuarios respecto a la prescripción de la ganancia (12). POGO propone un acercamiento que presenta la ganancia y la salida como características críticas en la prescripción (10). Esencialmente, el POGO es la regla de la mitad de la ganancia de Lybarger (3) con un factor de corrección en las bajas frecuencias para facilitar la mejor comprensión del habla (8). El POGO II modifica la ganancia cuando la pérdida auditiva es mayor de $65 \mathrm{~dB}$ (8). En la Tabla IV se presenta la fórmula y calculo del REIG según el POGO II.

La fórmula del POGO II también ofrece valores para determinar la máxima presión de salida del audífono en base a las medidas del UCL para 500 $\mathrm{Hz}, 1000 \mathrm{~Hz}$ y $2000 \mathrm{~Hz}$. Al igual que con el NAL-R, esta fórmula esta disponible en la mayoría de los módulos de adaptación y audioanalaizadores.

Tabla IV: Fórmula del POGO II para el cálculo del REIG propuesta por Schwartz, Lyergaard y Lunch (28).

\begin{tabular}{cccc}
\hline \hline & REIG & Saturación \\
\hline 250 & $1 / 2 \mathrm{HL}+1 / 2(\mathrm{HL} 65)$ & -10 & \\
500 & $1 / 2 \mathrm{HL}+1 / 2(\mathrm{HL} 65)$ & -5 & \\
1000 & $1 / 2 \mathrm{HL}+1 / 2(\mathrm{HL} 65)$ & & SSPL $90=$ \\
2000 & $1 / 2 \mathrm{HL}+1 / 2(\mathrm{HL} 65)$ & {$[(\mathrm{UCL} @ 500+1 \mathrm{~K}+$} \\
3000 & $1 / 2 \mathrm{HL}+1 / 2(\mathrm{HL} 65)$ & $2 \mathrm{~K}) / 3]+4$ \\
4000 & $1 / 2 \mathrm{HL}+1 / 2(\mathrm{HL} 65)$ & \\
\hline \hline
\end{tabular}

\section{Métodos de prescripción de la ganancia para audífonos no lineales}

\section{a) Desired Sensation Level Input/Output}

El DSL [i/o] es la actualización de la fórmulación original denominada simplemente DSL desarrollado en un principio para población pediátrica y audífonos lineales $(34,35)$. El propósito de Cornelisse et al. (7) al desarrollar el DSL fue obtener una fórmula de prescripción de la ganancia que permitiese individualizar el proceso de adaptación para la pérdida 
de un paciente determinado. En términos del propio autor, especificar de forma teórica la amplificación ideal para un rango de niveles de entrada para un individuo concreto. El DSL [i/o] se puede resumir como una serie de ecuaciones matemáticas (ver Tabla V) que describe la relación entre la señal de entrada y la señal de salida del audífono.

Tabla V: Fórmula del Desired Sensation Level Input/Output para el cálculo del REIG (7).

$$
\begin{aligned}
& \text { 1. } I<I_{\min }=(\text { Ganancia Lineal }): 0=0 0_{\min } I_{\min } \mid \\
& \text { 2. } I_{\min }<|<|_{\max }(\text { Compresión Lineal }): \\
& {\left[\begin{array}{c}
\mid-I_{\min } \\
I_{\max } I_{\min }
\end{array}\right] \times\left(0_{\max }-00_{\min }\right)+0_{\min } }
\end{aligned}
$$$$
\text { 3. } I>I_{\max } \text { (Limitación de Salida): } 0=0_{\max }
$$

El DSL [i/o] divide el rango dinámico de entrada en tres regiones: 1) niveles de entrada por debajo del umbral de compresión o $\mathrm{I}_{\min }$; 2) los niveles de entrada que exceden el umbral de compresión o $\mathrm{I}_{\max }$; 3) y el área entre estos dos limites. Para señales de entrada iguales o menores que $\mathrm{I}_{\min }$ se aplica amplificación lineal a la señal. Para señales iguales o mayores que $\mathrm{I}_{\max }$ la salida se limita a $\mathrm{O}_{\max }$ (limitación de salida). Para señales de entrada entre $I_{\min }$ e $I_{\max }$, la fórmula [i/o] puede ser aplicada tanto a un aparato con compresión de un canal o de varios canales. En el caso de audífonos lineales, la especificación de la ganancia por frecuencias y de salida máxima son suficientes para describir las características electroacústicas.

La prescripción de la ganancia en el DSL [i/o] es llevada acabo a partir de la audiometría del paciente en dB HL o mediante un SPL-O-GRAM en dB SPL. La audiometría tonal liminar puede llevarse a cabo mediante auriculares de inserción, auriculares TDH o en campo abierto. Independientemente del transductor utilizado, la audiometría vendrá siempre expresada en función de la edad y en dB SPL. El procedimiento ideal de recogida de la audiometría es mediante sonda microfónica en oído real y directamente en dB SPL. El DSL permite la introducción de valores individuales del paciente como la diferencia entre oído real y acoplador (RECD), la diferencia entre oído real y el dial del audiómetro (REDD) y la respuesta sin amplificar (REUR) (29). Todos estos parámetros nos permiten reducir la varianza error derivadas del uso de grupos control.

Una de las grandes ventajas de trabajar en $\mathrm{dB}$ SPL es que facilita el uso del promedio del espectro del habla o LTASS (30) y las medidas con sonda microfónica en oído real (31). La familiaridad del audioprotesista con estas medidas permite verificar la adaptación en función del LTASS (32). Esto permite predecir las zonas del espectro del habla que a pesar de la adaptación llevada a cabo pueden permanecer inaudibles lo cual puede ser de gran valor en la rehabilitación logopédica si la hubiere (32).
Adicionalmente podemos introducir información de los niveles de inconfort de sonoridad (LDL) para un paciente específico o recurrir a un baremo preestablecido a partir de los estudios de Pascoe (32). La máxima presión de salida se establece a partir de una desviación estándar de la media del LDL.

El DSL requiere información acerca del tipo de aparato (retroauricular, intracanal o intraauricular) ya que tiene en cuenta la localización del micrófono y esto afecta a la respuesta final en frecuencia del aparato.

Finalmente el DSL prescribe los valores de ganancia en función del tipo de compresión utilizada. Al adaptar un aparato no lineal el DSL nos sugiere obtener la curva de sonoridad. Esta puede obtenerse de forma individual o recurrir a los valores de referencia propios del programa. Cuando se escoge una compresión lineal (ratio de compresión fijo) los valores finales se obtienen a partir de los extremos de la función de incremento de sonoridad (umbrales y LDL). En ambos casos el DSL establece los valores de entrada propios de un rango dinámico normal dentro del rango dinámico del paciente (7).

Una vez introducida esta información el algoritmo del DSL [i/o] calcula los valores prescritos de la ganancia final en función de los niveles de entrada y sugiere las características electroacústicas del audífono (ganancia y ratios de compresión) para alcanzar el objetivo de amplificación propuesto. Sí el ratio de compresión excede 4:1 el programa nos avisa que un audífono no-lineal puede no ser apropiado para el paciente.

Además de dar los valores en dB SPL, el DSL [i/o] nos facilita los parámetros de la ganancia para acopladores de $2 \mathrm{~cm}^{3}$. Esto nos permite establecer comparaciones directas entre los valores ofrecidos por el fabricante y los prescritos por el DSL [i/o]. En caso de discrepancias estas medidas permiten la manipulación del audífono en el audioanalizador hasta alcanzar los valores prescritos.

El DSL [i/o] es una fórmula compleja que considera muchas variables en varias frecuencias de forma simultanea. El DSL [i/o] da al audioprotesista una gran flexibilidad ya que es posible obtener valores de RECD, REDD, REUR y la audiometría en dB SPL. Por otro lado es un procedimiento que sí no se dispone del tiempo necesario para llevar acabo la adaptación de forma individualizada el audioprotesista puede recurrir a valores promedios establecidos a partir de grupos control. De hecho la mayoría de los fabricantes ofrecen el DSL [i/o] en sus programas de adaptación sin necesidad de recurrir a todos los pasos propuestos por la formulación original.

\section{b) Independent Hearing Aid Fitting Forum (IHAFF)}

El IHAFF fue desarrollado por los miembros del Independetn Hearing Aid Fitting Forum (IHAFF) compuesto por investigadores, ingenieros y audiólogos clínicos con el objetivo común de desarrollar un protocolo estandarizado de adaptación protésica para ayudar en la selección y adaptación de audífonos no 
lineales y programables (33). El IHAFF mas que una fórmula es un método ya que propone como recoger los datos audiométricos, la selección apropiada del audífono y establece propuestas acerca de las medidas de validez de la adaptación (34). El marco teórico del IHAFF establece que un paciente tendrá un alto reconocimiento del habla en la medida en la que el audífono prescrito tenga la capacidad de restaurar la percepción de sonoridad normal en una banda de frecuencias lo más ancha posible (35).

Una de las principales diferencias entre el DSL [i/o] y el IHAFF son los datos audiométricos necesarios para obtener la prescripción final. Mientras que para el DSL [i/o] los requisitos mínimos son los valores de umbral audiométrico el IHAFF necesita además al menos de dos valores de la función de incremento de sonoridad del paciente. Estos valores se obtienen mediante el Contour Test que forma parte integral del IHAFF $(33,34,36)$. El Contour Test utiliza tonos modulados presentados al cliente en las frecuencias de $500 \mathrm{~Hz}$ y $3000 \mathrm{~Hz}$ (37). El cliente debe juzgar la sonoridad en una escala de siete categorías. El protocolo IHAFF utiliza un algoritmo denominado por los autores como VIOLA (Visualization of Input/Output Locator Algorithm) que facilita la manipulación de las variables implicadas en la selección del circuito del audífono (34, 37). El VIOLA calcula la relación entre la intensidad total de entrada para intensidades del habla suave, media y fuerte a la altura del micrófono y el juicio del usuario para los tonos modulados (33). Las respuestas se representan de forma individual según la categoría de sonoridad junto con una gráfica I/O indicando la entrada (de 30 a $90 \mathrm{~dB}$ en incrementos de $10 \mathrm{~dB}$ ) en el micrófono del audífono versus la salida del audífono medido en un acoplador $2 \mathrm{~cm}^{3} \mathrm{o}$ un HA-1.

En las últimas versiones del VIOLA es posible la introducción de diferentes combinaciones del total de la ganancia para una entrada de $40 \mathrm{~dB}$, características de salida y de compresión, salida máxima, salida total a $60 \mathrm{~dB}$ y características de la pendiente a las frecuencias en las que se han establecido los juicios de sonoridad. El audioprotesista puede valorar la relación entre la curva calculada de I/O y la ganancia prescrita.

\section{c) FIG 6}

A partir de la clasificación de las pérdidas auditivas establecidas por Killion y Fikret-Pasa (14) se desarrolló una fórmula basada en el concepto de sonoridad. El nombre de FIG6 surge de la figura número seis del artículo original de Killion y Fikret-Pasa (14). En la formulación original el FIG6 es un acercamiento que determina la ganancia en función de la respuesta en frecuencia del audífono $(38,39)$. El FIG6 puede ser utilizado para calcular la respuesta en frecuencia y la ganancia para sonidos de baja, media y alta intensidad. La fórmula del FIG6 puede observarse en la Tabla VI.
Tabla VI: Fórmula del FIG6 según Killion (38).

\begin{tabular}{|c|c|}
\hline \multirow{2}{*}{\multicolumn{2}{|c|}{ A. Ganancia para bajas intensidades }} \\
\hline & \\
\hline 2. $\mathrm{G}=\mathrm{HL}-20$ & De 20 a $60 \mathrm{~dB} \mathrm{HL}$ \\
\hline 3. $\mathrm{G}=\mathrm{HL}-20-0.5 \times(\mathrm{HL}-60)$ & $\mathrm{De} \mathrm{HL}>=60 \mathrm{~dB}$ \\
\hline \multicolumn{2}{|l|}{ B. Ganancia a MCL } \\
\hline 1. $G=0$ & De 0 a $20 \mathrm{~dB} \mathrm{HL}$ \\
\hline 2. $\mathrm{G}=0.6 \times(\mathrm{HL}-20)$ & De 20 a $60 \mathrm{~dB} \mathrm{HI}$ \\
\hline 3. $\mathrm{G}=0.8 \times \mathrm{HL}-23$ & $\mathrm{De} H \mathrm{H}>=60 \mathrm{~dB}$ \\
\hline \multicolumn{2}{|c|}{ C. Ganancia para altas intensidades } \\
\hline 1. $G=0$ & De 0 a $40 \mathrm{~dB} H \mathrm{H}$ \\
\hline 2. $\mathrm{G}=0.1 \times(\mathrm{HL}-40) \wedge 1.4$ & De $H \mathrm{~L}>=40 \mathrm{~dB}$ \\
\hline
\end{tabular}

El FIG6 se diferencia del IHAFF en el cálculo de los valores de la ganancia final ya que este se basa en baremos y no en datos individualizados. El FIG6 requiere únicamente de los datos audiométricos del paciente para el cálculo de la ganancia de inserción y de las respuestas en acoplador de $2 \mathrm{~cm}^{3}$ para los siguientes valores de entrada: 40, 65 y 95 dB SPL. Los valores de $2 \mathrm{~cm}^{3}$ están disponibles para audífonos retroauriculares, intrauriculares e intracanales. Las posibilidades de representación gráfica del FIG6 son muy amplias ya que nos da una gran variedad de opciones incluyendo los valores del REIG, valores en acoplador $2 \mathrm{~cm}^{3}$ y comparaciones entre los valores propuestos por el FIG 6 y el NAL-R (38).

El FIG 6, al igual que el DSL [i/o], calcula algunos parámetros que permiten a un audífono acercarse a los valores de ganancia prescritos. Estos parámetros son el ratio de compresión para las bandas de bajas y altas frecuencia, así como para altas y bajas intensidades de entrada (39). En caso de alcanzar un ratio de compresión por encima de 3:1 el software de adaptación del FIG6 recomienda considerar una amplificación lineal (38).

Ante pacientes con curvas de sonoridad alejadas de los grupos control empleado por el FIG6 será necesario llevar acabo algunos ajustes adicionales. Sin embargo, los valores prescritos por el FIG6 son adecuados como punto de partida de la adaptación y pueden ser útiles cuando solo se dispone de la audiometría tonal del paciente. Para complementar la adaptación mediante el FIG6 se pueden tomar las siguientes medidas adicionales; verificación del ajuste del audífono en el audioanalizador usando los valores prescritos para acoplador de $2 \mathrm{~cm}^{3}$, obtención de medidas de inserción en oído real para los diferentes valores de entrada y compararlos con los valores de ganancia calculados, introducción de los valores prescritos por el FIG6 en el audionalizador como método propio (esta opción está disponible en la mayoría de audioanalizadores) con el fin de facilitar las medidas en oído real y su comparación con los valores prescritos (38).

\section{d) NAL-NL1. National Acoustic Labs Non-Linear Version 1}

La filosofía general de los métodos desarrollados por el National Acoustic Labs es la prescripción de 
la ganancia mediante la amplificación ecualizada de la sonoridad de las distintas frecuencias del habla (40). Este criterio conlleva a una prescripción más acentuada de la ganancia en bajas frecuencias y a una normalización de la amplificación restringida a las frecuencias del habla.

El NAL-NL1 (40) es la versión para audífonos con compresión de los métodos de prescripción del National Acoustic Labs. Al igual que el NAL-R, las bajas, medias y altas frecuencias del espectro de la palabra hablada sé amplificadas con el criterio de alcanzar una sonoridad ecualizada. En NAL-NL1 prescribe para las frecuencias con peor audición menor ganancia que para aquellas con mejor audición. Para intensidades de entrada similares al promedio del habla conversacional (65 dB SPL) el NAL-NL1 prescribe valores de la ganancia muy parecidos a sus versiones anteriores. La principal diferencia de esta nueva versión la encontramos en la capacidad de la fórmula para dar valores distintos en función de diferentes valores de entrada.

Básicamente son dos los objetivos del NAL-NL1, en primer lugar trata de ecualizar antes que normalizar las frecuencias del habla prescribiendo menor ganancia en las bajas frecuencias. Segundo, tiene en cuenta la severidad de la pérdida de forma que en hipoacusias severas o profundas, el NAL-NL1 prescribe menos ganancia en altas frecuencias. En general, el NAL-NL1 prescribe la máxima ganancia en las frecuencias de transición de mejor a peor audición del audiograma; prescribiendo poca o ninguna amplificación en las frecuencias de peor audición.

Los fundamentos del NAL-NL1 se basan en estudios sobre individuos con perdida auditiva en los que se ha podido observar que estos poseen una menor capacidad para extraer información en la región de frecuencia en la que tienen la mayor parte de la perdida auditiva (40). Por ejemplo, en una perdida en pendiente que comienza a ser severa en altas frecuencias, la porción del habla correspondiente a estas frecuencias, no contribuye a mejorar la inteligibilidad del habla. En una perdida plana la mejora de la inteligibilidad del habla conlleva a la normalización de la sonoridad con un mayor énfasis en bajas frecuencias. En pérdida en altas frecuencias este énfasis disminuye la inteligibilidad. El objetivo del NAL-NL1 es prescribir una respuesta en frecuencia que mejore la inteligibilidad del habla manteniendo una sonoridad similar a la que percibe un normoyente. El NAL-NL1 no actúa sobre las frecuencias de forma individualizada sino que lo hace sobre el total del espectro del habla (40).

La fórmula de prescripción se desarrolló a partir de un modelo teórico de sonoridad y un modelo sobre la inteligibilidad del habla desarrollado a partir del Speech Inteligibility Index o Articulation Index (41). Este índice establece que porción del habla resulta audible para una pérdida determinada. Para poder incorporar este índice al NAL-NL1 fue necesario mejorar su exactitud mediante un factor de corrección al que denominaron Hearing Loss Desensitization (41). Este factor calcula para una intensi- dad de entrada, en función del rango del audiograma, una respuesta que mejore la inteligibilidad sin exceder un nivel de sonoridad que podríamos considerar como normal. Finalmente, el resultado es integrado en una fórmula que prescribe la ganancia en función de la frecuencia calculada a partir del umbral auditivo. La ganancia prescrita en cada frecuencia depende del umbral auditivo a esa frecuencia, el promedio del umbral en las frecuencias $500 \mathrm{~Hz}$, $1000 \mathrm{~Hz}$ y $2000 \mathrm{~Hz}$ y la intensidad total de la señal de entrada del habla.

\section{Conclusiones}

Cada uno de los métodos revisados consumen un tiempo que estará en función de la individualización del procedimiento. El incremento de la fiabilidad en la adaptación conlleva una mayor dedicación de tiempo ya que será necesario individualizar todas las variables que intervienen en la prescripción final de la ganancia. El IHAFF necesita de aproximadamente veinte minutos extras para obtener la curva de sonoridad. Posteriormente es necesario introducir esta información en el VIOLA en orden a prescribir los ajustes del audífono. El FIG6 no necesita de tiempo adicional y los ratios de compresión y los valores de ganancia final pueden obtenerse automáticamente. En el DSL [i/o] el audioprotesista puede decidir entre utilizar los valores individualizados de LDL y la curva de sonoridad o emplear baremos. El FIG6 y el DSL [i/o] no consumen mucho tiempo en la fase inicial pero si en la fase de validación y sobre todo en caso de que el paciente requiera algún ajuste adicional.

Los procedimientos revisados poseen bases teóricas diferentes y por lo tanto para un mismo paciente las prescripciones de la ganancia final son distintas. El IHAFF o el FIG6 tratan de restaurar la percepción de sonoridad normal, el DSL [i/o] trata de maximizar la audición en función del promedio del espectro de la palabra hablada y el NAL NL-1 trata de maximizar la información del habla a través de todas las frecuencias. No disponemos de comparativas que determinen la bondad de los métodos en función de las características audiológicas de los pacientes. En el momento de seleccionar un método u otro el audioprotesista debe tener en cuenta las características del paciente, la cantidad de tiempo disponible, los propios conocimientos y familiaridad con cada uno de los procedimientos.

La principal ventaja de estos métodos genéricos frente a los métodos desarrollados por los fabricantes son las mayores posibilidades de individualización de la adaptación y la posibilidad de prescribir parámetros de adaptación para una gran variedad de aparatos de distintas marcas. Los mayores inconvenientes, son el mayor tiempo dedicado a cada adaptación y la falta de información que estos métodos aportan acerca de parámetros como la frecuencia de cruce, el tiempo de ataque, los tiempos de recuperación o los puntos de inflexión, en el caso de los audífono no-lineales. 
En general, los métodos de prescripción de la ganancia genéricos aportan un mayor papel protagonista en la adaptación al audioprotesista ya que las decisiones a tomar son mayores permitiendo aproximar la adaptación de una forma mucho más exacta a las necesidades del paciente. Así mismo, dotan al audioprotesista de una hipótesis de trabajo establecida a través de los valores de la ganancia prescrita que permitirán integrar la adaptación en el proceso de validación a través del cual falsear la bondad de la hipótesis y compara en que medida se alcanza el referente ideal propuesto por el método adoptado. Mediante este proceso, el audioprotesista obtiene una mayor racionalización del trabajo y un mayor control sobre la adaptación.

\section{Bibliografía}

1. Carhart R. (1946). Tests for selection of hearing aids. Laryngoscope. 56:780-94.

2. Watson y Knudsen V. (1940).Selective amplification in hearing aids. J Acoust Soc of Am. 18:401-8.

3. Lybarger S. (1944). Method of fitting hearing aids. U.S. Patent Applications S.N. 543,278 .

4. Davis H, Hudgins C, Marquis R, et al. (1946). The selection of hearing aids. Laryngoscope. 56:85-163.

5. Teter D. (1989). Keynote address: Hearing aids and aural rehabilitation. Academy of Rehabilitation in Audiology, Summer Institute. Austin, TX.

6. Berger KW, Hagberg EN, Rane RL. (1988). Prescription of hearing aids: Rationale, Procedure and Results. 5th Edition, Herald Publishing, Kent $\mathrm{OH}$.

\section{Cornelisse LE, Seewald RC, Jamieson} DG. (1995). The input output fórmula: A theoretical approach to the fitting of personal amplification devices. J Acoust Soc Am. 97:1854-64.

8. Hawkins D. (1992). Prescriptive approaches to selection of gain and frequency response, In: Mueller HG, Hawkins DB, Northern JL, editors. Probe tube microphone measurements: hearing aid selection and assessment. San Diego, CA: Singular Publishing.

9. Byrne D. (1993). Hearing aid gain and frequency response selection strategies. In: Studebaker GA, Hochberg I, editors. Acoustic factors affecting hearing aid performance. Boston: Allyn and Bacon.
10. McCandless G. (1994). Hearing aid fórmulae and their application, In: Sandlin R, editor. Hearing aid handbook: volume I theoretical and technical considerations. San Diego, CA: College-Hill Press.

11. Kießling J. Hearing aid fitting and verification strategies . (citado 1 de agosto de 2001). Disponible en internet en: http://www.crsamplifon.com/OtoAcademy/Fi lePdf/Oto_Jürgen.pdf

12. McCandless G. y Lyregaard P. (1983). Prescription of gain and output (POGO) for hearing aids. Hear Instr. 34:16-21.

13. Byrne D, Dillion H. (1986). The National Acoustic Laboratories (NAL) new procedure for selecting the gain and frequency response of a hearing aid. Ear Hear. 7:25765.

14. Killion M. y Fikret-Pasa. (1993). The three types of sensorineural hearing loss: loudness and intelligibility consideration. Hear J.46:31-6.

15. Seewald RC. (1995). The Desired Sensation Level (DSL) method for hearing aid fitting in infants and children. Phonak Focus Nr. 20.

16. Dillon H, Katsch R, Byrne D, Ching T, Keidser G, Brewer S. (1998). The NALNL1 prescription procedure for non-linear hearing aids. NAL Annual Report 1997/98, 4-8.

17. Allen JB, Hall JL, Jeng PS. (1990) Loudness growth in 1/2-octave bands (LGOB) A procedure for the assessment of loudness. J Acoust Soc Am 88, 745-753.

18. Kießling J. (1997) Versorgung mit Hörgeräten. In: Kießling J, Kollmeier B, Diller G: Versorgung und Rehabilitation mit Hörgeräten. Thieme Verlag Stuttgart, 49-109.

19. Haubold J. (1996). Zukünftige Hörgeräteanpassung aus der Sicht der Hörgeräteakustik. 8. Multidisziplinäres Kolloquium der Geers-Stiftung, Band 11, 147163.

20. Moore BCJ, Alcantara JI, Glasberg BR. (1998). Development and evaluation of a procedure for fitting multi-channel compression hearing aids. Br J Audio/ 32 (1998) 177-195.

21. Pastoors AD, Gebhart TM, Kiessling J. (1999). A fitting strategy for digital hearing 
aids based on loudness and sound quality. Proceedings of 4th European Conference on Audiology, Scand Audiol Suppl. (in press).

22. Schum, D. (1996). Adaptive Speech Alignment: A new fitting rationale made possible by DSP. The Hearing Journal, 49:5, 25-30.

23. Ludvigsen C. y Topholm J. (1997). Fitting a wide dynamic range hearing instrument using real ear threshold data: a new strategy. Hear Rev: High Performance Solutions 2:37-39.

24. Byrne D. y Tonnisson W. (1976). Selecting gain of hearing aids for persons with sensorineural hearing impairments. Scand Audiol. 5:51-9.

25. Mueller HG. (1992). Individualizing the ordering of custom hearing aids, In: Mueller HG, Hawkins DB, Northern JL, editors. Probe-microphone measurements: hearing aid selection and assessment, San Diego, $\mathrm{CA}$ : Singular Publications.

26. Bryne D, Parkinson A, Newell P. (1990). Hearing aid gain and frequency response requirements for the severely/profoundly hearing impaired. Ear Hear 1990;11:40-9.

27. Byrne D, Parkinson A, Newell P. (1991) Modified hearing aid selection procedures for severe/profound hearing losses. In: Studebaker G, Bess F, Beck L, editors. The Vanderbilt hearing aid report II. Parton, MD: York Press; 1991. p. 295-300.

28. Schwartz D, Lyregaard $P$, Lundh $P$. (1988). Hearing aid selection for severe-toprofound hearing loss. Hear J. 41:13-7.

29. Mueller H.B. (1995). Prescriptive hearing aid fitting, current issues in amplification. St. Louis, MO: Washington University.

30. Delgado J. y Zenker F. (2002). El promedio del espectro del habla: Fundamentos y aplicaciones clínicas [en-linea]. Auditio: Revista electrónica de audiología. 1(3)4144.

<http://www.auditio.com/revista/pdf/vol1/3/ 010303.pdf>

31. Zenker F. y Barajas J. (1999). Adaptación de audífonos en función del promedio del espectro de la palabra hablada. Estudio de un caso único. En: Logopedia escolar y clínica. Últimos avances en Evaluación e Intervención. Editor: José Domingo Martín
Espino, Madrid, Editorial CEPE, pp. 329336.

32. Pascoe, D.L. (1988). Clinical measurements of the auditory dynamic range and their relation to formulas for hearing aid gain. In J. Hensen (Ed.), Hearing aid fitting: Theoretical and practical views (pp. 129152). Proceedings of the 13th Danavox Symposium, Copenhagen.

33. Van Vliet D. (1995). Determining contour loudness judgements. Hear Instr. 46(March):30.

34. Cox R. (1995). Using loudness data for hearing aid selection: the IHAFF approach. Hear J.48:2, 10, 39-44.

35. IHAFF. (1994). A comprehensive hearing aid fitting protocol, distributed at Jackson Hole Rendezvous. Jackson, WY.

36. Valente M. y Van Vliet D.(1997). The independent hearing aid fitting forum (IHAFF) protocol. Trends in Amplification, 2(1),6-35.

37. Cox R, Goff CM, Martin SE, McLoud LL. (1994). The contour test: normative data, Presented at the AAA Convention. Richmond, VA.

38. Killion M. (1994). FIG6. Jackson Hole Rendezvous. Jackson, WY.

39. Sullivan R. (1988). Probe tube microphone placement near the tympanic membrane. Hear Instr. 39:43-4, 60.

40. Byrne D, Dillon H, Ching T, Katsch R \& Keidser G. (2001). NAL-NL1 procedure for fitting nonlinear hearing aids: Characteristics and comparisons with other fitting methods. Jour AAA, 12 (1): 37-51.

41. Rankovic CM. (1991). An application of the articulation index to hearing aid fitting. Journal of Speech and Hearing Research. 34:391-402.

Recibido el 13 de Julio del 2002.

Aceptado el 28 de Agosto del 2002.

Publicado (on-line) 1 de Octubre del 2002.

Contacto con el autor: Franz Zenker. Clínica Barajas. C/ Pérez de Rozas 8. 38004 Santa Cruz de Tenerife. Islas Canarias. España. Tel: +34 922275488 Fax: +34 922 270364 E-mail: zenker@clinicabarajas.com

Para citar este artículo: Zenker, F. La prescripción de la ganancia en la adaptación audioprotésica. [en-línea]. Auditio: Revista electrónica de audiología. 1 Octubre 2002, vol. 1(3), pp. 45-52. <http://www.auditio.com/revista/pdf/vol1/3/ 010304.pdf> 